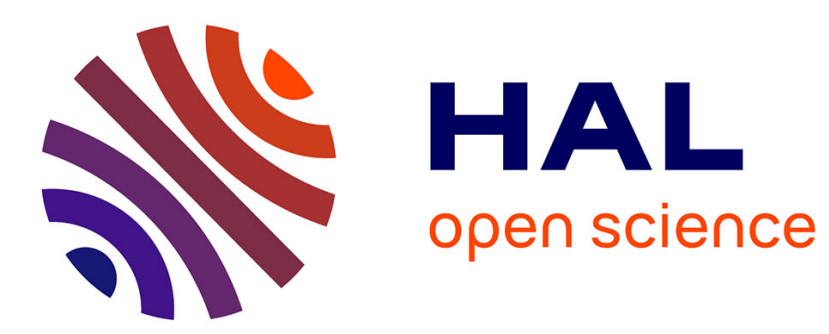

\title{
Typical Aspects of the Microwave Noise Performance of HEMTs at Decreasing Temperatures
}

\author{
A. Caddemi, M. Sannino
}

\section{To cite this version:}

A. Caddemi, M. Sannino. Typical Aspects of the Microwave Noise Performance of HEMTs at Decreasing Temperatures. Journal de Physique IV Proceedings, 1996, 06 (C3), pp.C3-151-C3-156. 10.1051/jp4:1996323 . jpa-00254241

\section{HAL Id: jpa-00254241 https://hal.science/jpa-00254241}

Submitted on 1 Jan 1996

HAL is a multi-disciplinary open access archive for the deposit and dissemination of scientific research documents, whether they are published or not. The documents may come from teaching and research institutions in France or abroad, or from public or private research centers.
L'archive ouverte pluridisciplinaire HAL, est destinée au dépôt et à la diffusion de documents scientifiques de niveau recherche, publiés ou non, émanant des établissements d'enseignement et de recherche français ou étrangers, des laboratoires publics ou privés. 


\title{
Typical Aspects of the Microwave Noise Performance of HEMTs at Decreasing Temperatures
}

\author{
A. Caddemi and M. Sannino
}

Dipartimento di Ingegneria Elettrica, Università di Palermo, Laboratorio di Electtronica delle Microonde, Viale delle Scienze, 90128, Palermo, Italy

\begin{abstract}
In analog signal processing at microwave frequencies the noise performance of active devices is of fundamental importance for the accurate design of low-noise amplifiers. To this aim, the determination of the four noise parameters $F_{0}, \Gamma_{O}$ (complex variable) and $R_{n}$ has to be accomplished together with the usual scattering parameter measurcments vs. frequency. In addition, the dependence of the device performance $v$ s. temperature is of interest for circuit applications characterized by harsh environmental conditions. In this work the noise behavior of high electron mobility transistors has been investigated by means of measurements and modeling in the $2-18 \mathrm{GHz}$ frequency range and as a function of the decreasing temperature over the $-50-+20^{\circ} \mathrm{C}$ range. The most interesting aspects of the observed variations occurred with temperature are explained on the basis of the device equivalent circuit.
\end{abstract}

\section{INTRODUCTION}

Knowledge of the noise behavior of active devices is the main concern in designing low-noise amplifiers (LNA) for analog signal processing in the microwave frequency range $(1-30 \mathrm{GHz})$.

At present, conventional GaAs and pseudomorphic high electron mobility transistors (namely, HEM'T and pHEM'T) exhibit the best noise and gain performance among solid-state devices operating up to the millimeter wave range. Due to this features, they are widely used for the realization of LNA's to be employed in applications such as satellite communication, radio astronomy, remote sensing, and so on, where maximum signal sensitivity is strictly required.

To the aim of obtaining a complete characterization of the active device under test (DUT) we need to measure both the scattering and the noise parameters vs. frequency. The results of the characterization can either be employed as a black-box model of the DUT or be used for the extraction of an equivalent circuit model which is a more accurate and flexible tool for describing the DUT performance in CAD applications.

In this work, the microwave performance of different series of HEMT has been investigated as a function of frequency $(2-18 \mathrm{GHz})$ and decreasing temperature (from $20^{\circ} \mathrm{C}$ to $-50^{\circ} \mathrm{C}, 10^{\circ} \mathrm{C}$ step) for assessing the most typical aspects of such devices from the noise viewpoint.

The devices have been tested at each temperature in terms of scattering parameters and noise figure $F_{50}$ in input matched conditions (i.e. @ standard source impedance of $50 \Omega$ ) at the bias conditions suggested by the manufacturer for the best low-noise performance. In addition, at room temperature we performed a complete characterization of the devices by determining all the noise, gain and scattering parameters with a noise figure measuring system only, whose relevant measurement procedures and data processing techniques have been totally developed in our Lab [1].

A model extraction has then been accomplished in terms of an equivalent electrical network for computing the four noise parameters $F_{o}, \Gamma_{O}$ (complex variable) and $R_{n} v s$. temperature by model analysis. The most interesting aspects of the observed variations occurred with temperature have been explained on the basis of the noise behavior of device model which has previously shown (at room temperature) a very good degree of reliability in predicting the actual device performance.

This work was supported by Italian Space Agency (ASI), National Research Council (CNR) and Ministry of University, Science and Technology Research (MURST). 


\section{DEVICE CHARACTERIZATION}

At microwave frequencies, the noise parameters usually determined are represented by the minimum noise figure $F_{O}$, the relevant optimum (complex) value $\Gamma_{O}$ of the input termination reflection coefficient $\Gamma_{S}$ at which $F_{O}$ is located, and the equivalent noise resistance $R_{n}$ which is a measure of the noise figure degradation upon departure of $\Gamma_{S}$ from its optimum value. The noise equation linking the above parameters is:

$$
F\left(\Gamma_{S}\right)=F_{o}+4 R_{n} \frac{\left|\Gamma_{s}-\Gamma_{o}\right|^{2}}{\left|1+\Gamma_{o}^{2}\right|\left(1-\left|\Gamma_{s}\right|^{2}\right)}
$$

Basically, the determination of the above noise parameters is accomplished by source-pull measurements, i.e. by measuring the noise figure value at properly selected input reflection coeffiecients $\Gamma_{\mathrm{S}}$ (more than four, for redundancy) and by processing data through eq.(1).

In our Lab an original and yet well-assessed tecnique has been developed several years ago which allows for determining either the noise parameters and the scattering parameters of the DUT from all and only noise data. The scattering parameters, when determined by our system, are not measured directly by a network analyzer (see [1] and references therein).

The system is now operating in the updated version over the $1-40 \mathrm{GHz}$ frequency range, vs. bias and temperature, for the complete characterization of low-noise devices in packaged and in chip form. A block diagram is reported in Fig.1.

As far as the present work is concerned, two different HEMT series (CFB001-03 by Celeritek, TC2623 by Thomson) have been characterized first at room temperature in terms of noise, gain and scattering parameters by means of our system in the $6-14 \mathrm{GHz}$ frequency range. As a second step, their scattering parameters were measured from 2 to $18 \mathrm{GHz}$ by use of the automatic network analyzer with the DUT placed in a thermostatic chamber to control its ambient temperature over the $-50-+20{ }^{\circ} \mathrm{C}$ range at $10^{\circ} \mathrm{C}$ steps. In the same operating conditions, we employed a commercial noise figure meter for performing noise figure measurements at the fixed condition of $\Gamma_{S}=0$ (i.e., $50 \Omega$ impedance), which is usually referred to as $F_{50}$. The measured values of $F_{50}$ have then been used for the simplified determination of a noisy model of HEMTs, as discussed in the modeling section of this paper.

\section{MODELING PROCEDURE}

The equivalent circuit model has been determined for each device series by fitting the measured S-parameters vs. temperature. The model extraction has been accomplished by following the subsequent steps: 1) fixing the basic circuit topology which includes package parasitics; 2) applying a decomposition approach for the optimization of the circuit variables at room temperature; 3 ) adding knowledge of the behavior of the chip model elements to provide physical consistency and additional contraints for the optimization at lower temperatures.

The basic structure obtained for the given transistor series is sketched in Fig.2; the associated element values vary according to each HEMT set and the decreasing temperature.

The circuit elements which mainly affect the microwave performance of the device are the transconductance $g_{m}$, the gate-to-source capacitance $C_{g s}$ and the output resistance $R_{d s}$. Their value trends are in accordance with the physics-based behavior.

The complete noise performance of the device has been reproduced by associating to its equivalent circuit a simple noise model which employs two uncorrelated equivalent noise temperatures (namely, $T_{c h}$ and $T_{d}$ ) associated with $R_{c h}$ and $R_{d s}$, desides the thermal noise due to the remaining lossy elements of the circuit model. More practically, since $T_{c h}$ has been found to approach the ambient temperature for HEMTs, this model reduces to the single frequency-independent factor $T_{d}$ whose value can be derived by fitting either the optimum noise figure $F_{0}$ or the $F_{50}$ noise figure. Common values of this fitting factor lie within the 1000 $5000 \mathrm{~K}$ range and decrease with either decreasing temperature and decreasing noise figure values.

At each ambient temperature we determined the relevant $T_{d}$ value and properly set the other resistor temperatures. By model analysis, we then computed the complete noise performance in 
terms of the four parameters. We had previously tested the effectiveness of this procedure by comparing the simulated noise parameters with the measured ones deriving from a complete characterization at room temperature $\left(20^{\circ} \mathrm{C}\right)$. The results of this comparison showed that we can get noise parameter values close within a $10 \%$ difference which supports the reliability of this method [3].

\section{RESULTS}

Sample plots showing the measured scattering parameters and the $F_{50}$ noise figure vs. frequency and temperature are reported in Figs.3-5 for the TC2623 series by reporting the statistically averaged behavior. It is clearly seen that the performance of the devices in terms of scattering parameters is not much changed, which is to be expected due to the reduced temperature lag $\left(70^{\circ} \mathrm{C}\right)$. Substantial alterations of these devices can be observed for temperature ranging down to $-100^{\circ} \mathrm{C}$. The other parameters show only subtle changes which, however, affect the device modeling.

However, slightly pronounced effects are observed for $\left|S_{21}\right|$ (i.e. the device gain) which exhibits a clear trend for increasing upon cooling down the device, and $\left|S_{22}\right|$ which decreases with temperature. The behavior of these parameters is directly related to the device channel properties which are affected by temperature changes. As a consequence on the model element values, we note an increase of the transconductance $\mathrm{g}_{\mathrm{m}}$, a decrease of the output resistance $R_{\mathrm{ds}}$ and an increase of the output capacitances $\mathrm{C}_{\mathrm{ds}}$ and $\mathrm{C}_{\mathrm{pd}}$. In addition, the expected increase of the cutoff frequency $f_{T}=g_{m} / 2 \pi C_{g s}$ as the temperature lowers is due to either the increase of $g_{m}$ and a decrease of the values of $\mathrm{Cgs}_{\mathrm{gs}}$.

As far as the noise figure $\mathrm{F}_{50}$ is concerned, the measured data show that it decreases by lowering the temperature and the slope of the relevant curves vanishes. As an example, the performance of $F_{50}$ at $-40^{\circ} \mathrm{C}$ is quite flat with values below $1 \mathrm{~dB}$ over the entire measured frequency range $(6$ to $16 \mathrm{GHz}$ ). Such a behavior is almost ideal for an active device to be employed in a low-noise wideband amplifier since it would allow for obtaining a very low noise figure without any tuning network at the device input port.

On the basis of the measured values of $F_{50}$ we derived the noise fitting factor $T_{d}$ and employed the circuit models of the two HEMT sets for deriving the complete noise performance at decreasing temperatures.

For the sake of comparison with the model performance at different temperatures, the complete noise parameters relevant to all devices of the CFBO01-03 series at room temperature is shown in Fig.6a-d. By model analysis we have then derived the noise parameters upon lowering the noise temperatures of the resistive elements and varying accordingly the values of the circuit elements. By analyzing the latter HEMT type, we note that the noise performance exhibits a marked change in the behavior of $1 \Gamma_{\mathrm{o}}$ with respect to that observed at $20^{\circ} \mathrm{C}$. The behavior of $\left|\Gamma_{\mathrm{o}}\right|$ is shown in Fig. 7 and the substantial variation occurring with the temperature is represented by its trend for becoming higher than $\left|S_{11}\right|$ in the middle of the frequency range (about 6 to $14 \mathrm{GHz}$ ). The reason of this variation has been investigated by model analysis to the aim of separating the contributions due to the noise temperatures of the resistive elements from those originated by the different value sets of the model elements. It has been found that the main cause of this performance of $\left|\Gamma_{\mathrm{o}}\right|$ at decreasing temperatures is the change in the circuit element values since the noise temperatures play a minor role over the observed value ranges [5].

As far as the phase of $\Gamma_{0}$ is concerned, we observed that its performance is not much affected by the temperature, except for slight variations at the higher frequencies.

The behavior of $F_{o}$ is linear vs. frequency, even at low temperatures, but its slope and its values decrease markedly as it occurs to $F_{50}$. The slope reduces to $0.04 \mathrm{~dB} / \mathrm{GHz}$ at $-50{ }^{\circ} \mathrm{C}$ which is $50 \%$ of its value at $20^{\circ} \mathrm{C}$, and the value of $F_{O}$ at $18 \mathrm{GHz}$ is $0.7 \mathrm{~dB}$.

Finally, we noted that the curves of the equivalent noise resistance $R_{n}$, shown in Fig.8, broaden with frequency by lowering the temperature. Also, it decreases proportionally thus reaching values less than $1 \Omega$ in the middle frequency range.

While the performance of $F_{0}$ is directly affected by the decrease of the model noise temperatures (mainly $T_{d}$ ), the performance of $F_{50}$ is determined by either the lower noise temperatures and the "combined" influence of $R_{n}$ and $\Gamma_{O}$ which are heavily affected by the 
change of the model element values with the temperature. As an overall result, the noise performance of the device markedly improves upon decreasing the ambient temperature.

The model prediction will be compared with the results of the complete noise characterization vs. temperature which is currently in progress.

\section{CONCLUDING REMARKS}

In this work, the microwave performance of different series of HEMT has been investigated as a function of frequency $\left(2-18 \mathrm{GHz}\right.$ ) and decreasing temperature (from $20^{\circ} \mathrm{C}$ to $-50^{\circ} \mathrm{C}, 10^{\circ} \mathrm{C}$ step) for assessing the most typical aspects of such devices from the noise viewpoint.

The devices have been tested at each temperature in terms of scattering parameters and noise figure $\mathrm{F}_{50}$ in input matched conditions. In addition, at room temperature we performed a complete characterization of the devices by determining all the noise, gain and scattering parameters with an original noise figure measuring system only.

A model extraction has then been accomplished in terms of a noisy equivalent electrical network for computing the four noise parameters vs. temperature by model analysis. The most interesting aspects of the observed variations occurred with temperature have been presented and evaluated. Knowledge of the changes in the device noise parameters is of basic importance in accomplishing the accurate design of low-noise amplifiers for applications in unfriendly environmental conditions where a high-level performance of the receiver has to be guaranteed.

\section{References}

[1] G. Martines and M. Sannino, "The determination of the noise, gain and scattering parameters of microwave transistors (HEMTs) using only an automatic noise figure testset", IEEE Trans. Microwave Theory Tech., vol. MTT-42 (1994), 1105-1113.

[2] M.Pospieszalski, "Modeling of noise parameters of MESFETs and MODFETs and their frequency and temperature dependence", IEEE Trans. Microwave Theory Tech., vol. MTT-37 (1989), 1340-1350.

[3] A. Caddemi, A. Di Paola, M. Sannino, "Complete characterization of low-noise devices at microwave frequencies: two alternative procedures for HEMTs", Proceedings of the 46th Conference of the Automatic Radio Frequency Techniques Group (ARFTG), Phoenix, USA, Dec. 1995.

[4] A. Caddemi, M. Sannino, "On the performance of the noise parameters of HEMTs at microwave frequencies", Proceedings of the 2nd IEEE International Conference on Electronics, Circuits and Systems, pp.124-131, Amman, Jordan, Dec. 1995.

[5] A. Caddemi, M. Sannino and F. Di Prima (student), "Performance of the optimum noise source reflection coefficient $\left(\Gamma_{0}\right)$ vs. temperature in microwave HEMTs by model analysis", Proceedings of the 18th International Semiconductor Conference, pp.499502, Sinaia, Romania, Oct. 1995.

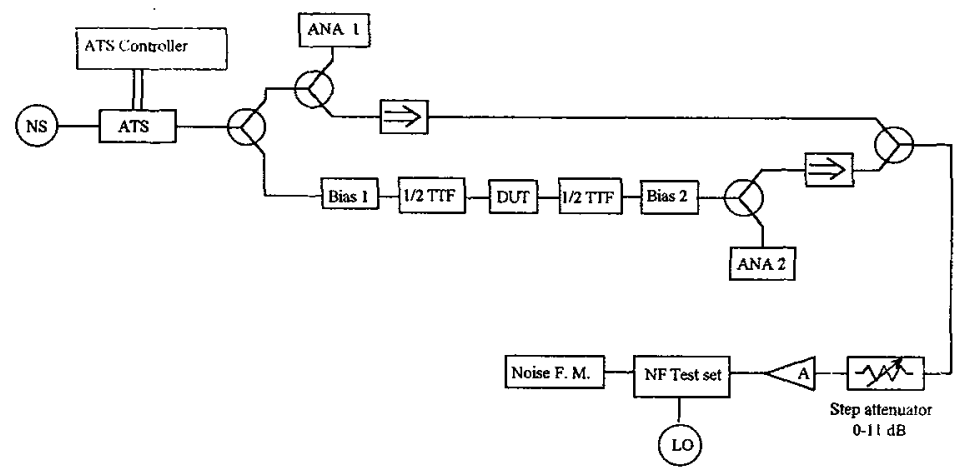

Figure 1: Block diagram of the noise figure measuring system employed for the complete characterization of low-noise active devices 


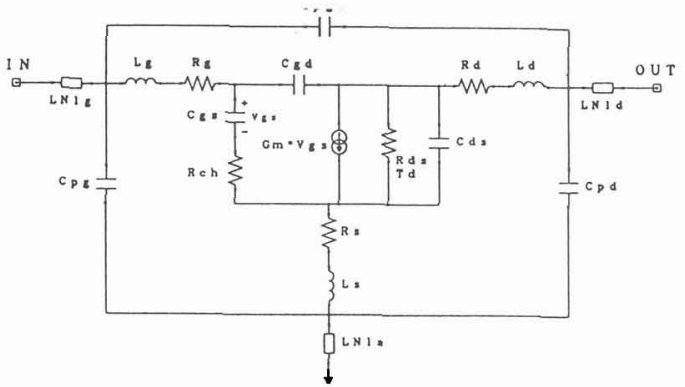

Figure 2: Equivalent circuit model extracted for the HEMT series (CFBO01-03, by Celeritek, and TC2623 by Thomson) characterized vs. frequency and temperature

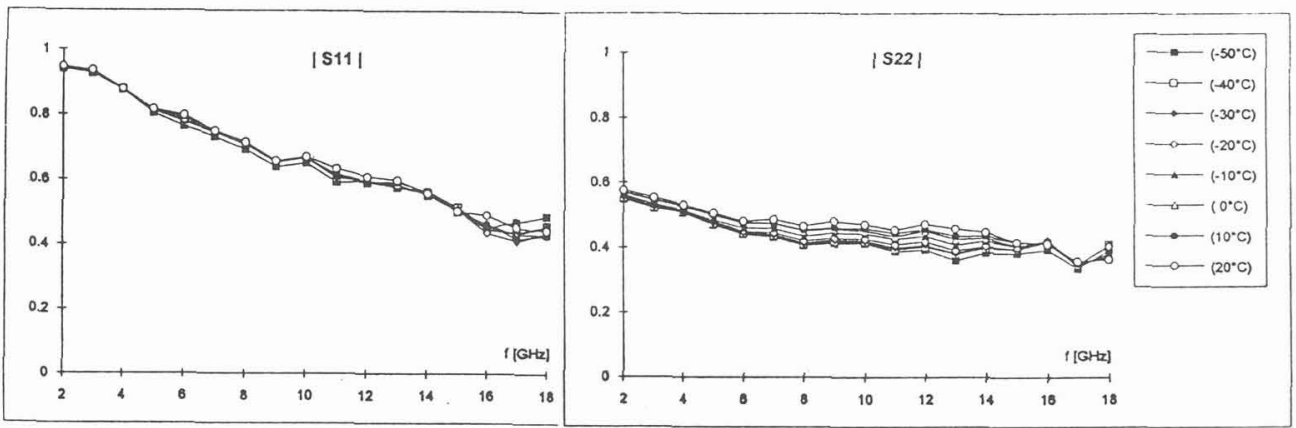

Figure 3: Measured $\left|S_{11}\right|$ and $\left|S_{22}\right|$ parameters (average device performance) as a function of frequency and temperature for the TC2623 series

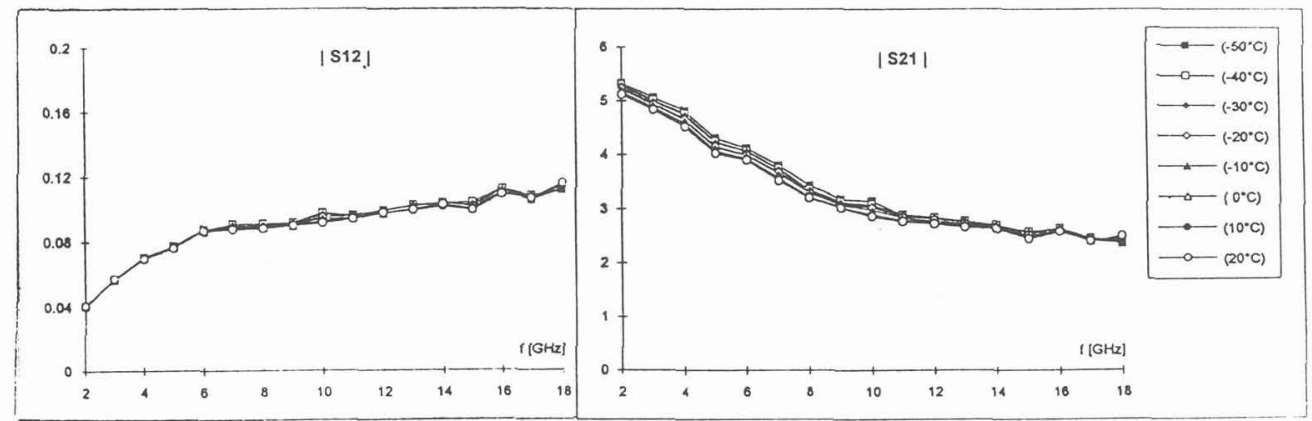

Figure 4: Measured $\left|S_{12}\right|$ and $\left|S_{21}\right|$ parameters (average device performance) as a function of frequency and temperature for the TC2623 series

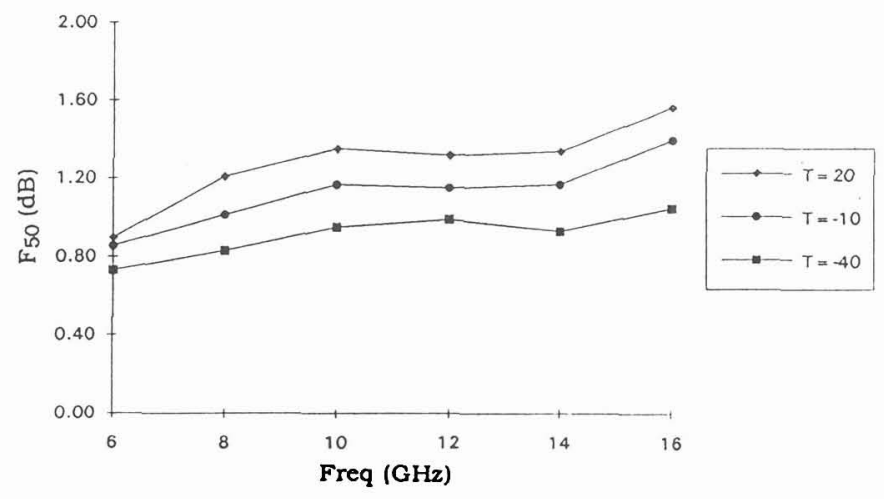

Figure 5: Measured performance of the noise figure F50 (typical device of the TC2623 series)as a function of frequency and temperature $T$ 


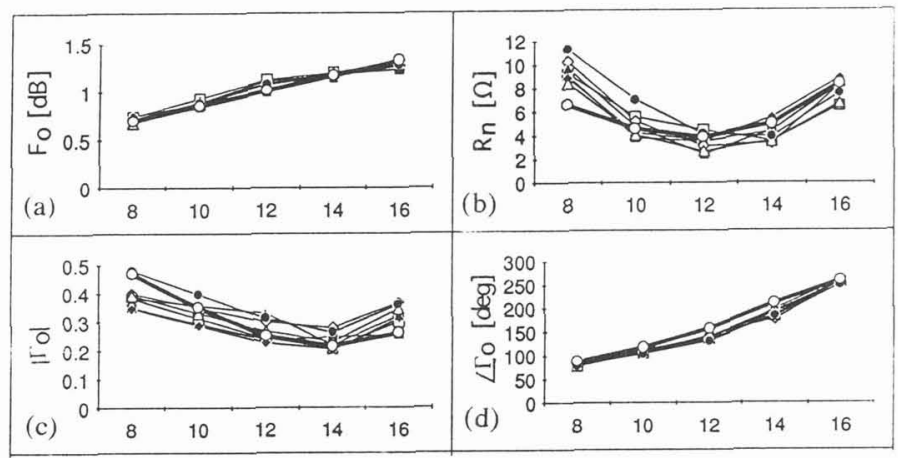

Figure 6: Measured noise parameters of the FBí()1-(1)3 HEMT senes at rexm temperature: a) minimun noise figure $\left.F_{0}(\mathrm{~dB}) ; b\right)$ magnitude of the optimum noise reflection coefficient $\Gamma_{\mathrm{O}} ; \mathrm{c}$ ) angle of the optimum noise reflection cocfficient $\Gamma_{0}$; d) equivalent noise resistance $R_{n}(\Omega)$

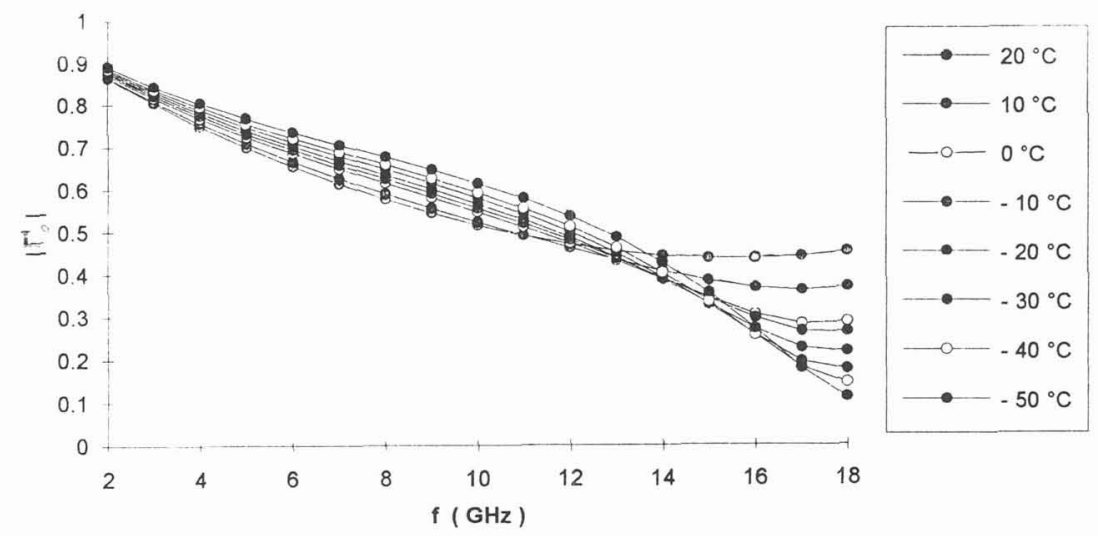

Figure 7: Computed performance of $1 \Gamma_{0} \mid \mathrm{vs}$. frequency at decreasing temperatures(CFBO0)1-()3 series)

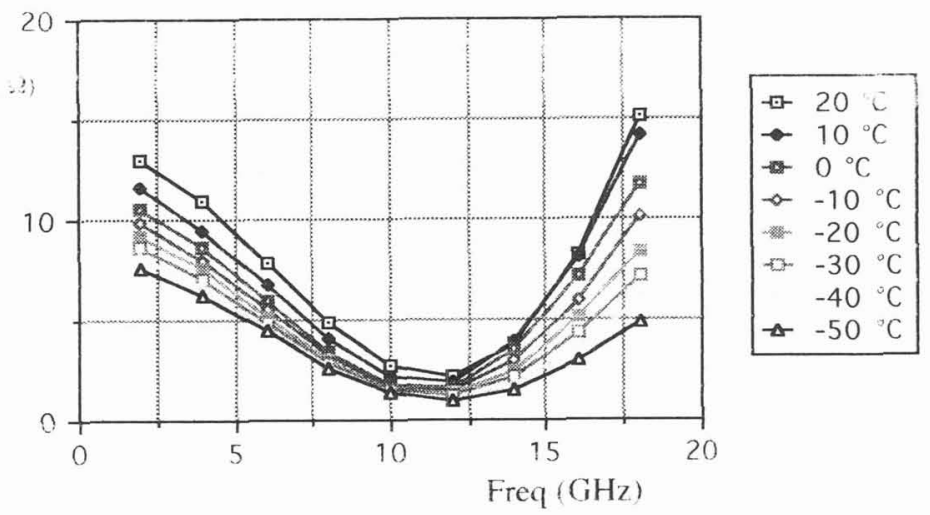

Figure 8: Pertormance of the equivalent noise resistance $R_{n}$ modeled is. frequency at decreasing temperatures (model of the CFB(0)1-(1)3 series) 